\title{
Long non-coding RNA CIR inhibits chondrogenic differentiation of mesenchymal stem cells by epigenetically suppressing ATOH8 via methyltransferase EZH2
}

\author{
Feng Liu ${ }^{1,2}$, De-Ye Song ${ }^{1}$, Jun Huang ${ }^{1}$, Hong-Qi Yang ${ }^{2}$, Di You ${ }^{1}$ and Jiang-Dong Ni ${ }^{1 *}$
}

\begin{abstract}
Background: Osteoarthritis $(\mathrm{OA})$ is the most common articular disorder, leading to joint malfunction and disability. Although the incidence of $O A$ is increasing globally, the treatment of $O A$ is very limited. LncRNA CIR has been implicated in OA through unclear mechanisms. Here, we investigated the role of IncRNA CIR in chondrogenic differentiation.
\end{abstract}

Methods: Human umbilical-cord-derived mesenchymal stem cells (hUC-MSCs) were obtained from human umbilical cords. Flow cytometry was used to analyze the surface markers of hUC-MSCs. Various culture conditions and corresponding staining assays were employed to assess the differentiation abilities of hUC-MSC. qRT-PCR, western blot, and immunostaining were used to measure expression levels of related genes and proteins such as IncRNA CIR, ATOH8, $\mathrm{EZH} 2$, and H3K27me3. RNA immunoprecipitation assay, biotin pull-down, and chromatin immunoprecipitaion assay were performed to analyze the interactions of IncRNA CIR, EZH2, H3K27me3 and ATOH8 promoter.

Results: hUC-MSCs exhibited MSCs features and could differentiate into chondrocytes under specific conditions. LnCRNA CIR was downregulated while ATOH8 was upregulated during the chondrogenic differentiation of hUC-MSCs. Knockdown IncRNA CIR or overexpression of ATOH8 promoted chondrogenic differentiation. Further, IncRNA CIR bound to $\mathrm{EZH} 2$ and repressed ATOH8 expression via EZH2-mediated H3K27me3, which promotes the methylation of ATOH8. Inhibition of ATOH8 reversed the effects of knockdown IncRNA CIR on chondrogenic differentiation.

Conclusion: LncRNA CIR suppresses chondrogenic differentiation of hUC-MSCs. Mechanistically, IncRNA CIR could inhibit ATOH8 expression that functions to promote chondrogenic differentiation through EZH2-mediated epigenetic modifications.

Keywords: LncRNA CIR, ATOH8, Chondrogenic differentiation, EZH2, hUC-MSC, Osteoarthritis

\section{Introduction}

Osteoarthritis (OA) is a very common but complex chronic degenerative disease characterized by

\footnotetext{
*Correspondence: nijiangdong@csu.deu.cn

${ }^{1}$ Department of Orthopedics, The Second Xiangya Hospital of Central

South University, No.139 Renminzhong Road, Changsha 410011, Hunan, People's Republic of China

Full list of author information is available at the end of the article
}

degradations of articular cartilage, thickening of the joint capsule, and the formation of osteophyte, leading to the progressive loss of joint mobility and function (Mehl et al. 2018). It is estimated to be the fourth leading cause of disability in Asia and the incidence of OA worldwide is on a steady rise (Vina and Kwoh 2018; Fransen 2011). Currently, there are no effective treatments that can restore the function of joint (Vargas Negrin et al. 2014). 
The pathogenesis of OA is complicated and multifactorial, which is mainly associated with age-related loss of homeostatic balance (Martel-Pelletier 2016). Articular chondrocyte, the only cell type in the articular cartilage, which has the function of maintaining tissue homeostasis (Carballo et al. 2017). During OA development, processes like inflammatory responses and the release of reactive oxygen species (ROS) induce remarkable changes to articular chondrocytes that result in their malfunction and eventually death (Caldwell and Wang 2015; Lepetsos and Papavassiliou 2016). Therefore, understanding the molecular mechanisms of chondrocyte homeostasis is very important for the development of new therapeutic avenues.

Emerging evidence has shown that mesenchymal stem cells (MSCs) could be used for cartilage repair in that they are multipotent stromal cells and can differentiate into varieties of cell types, including chondrocytes (Chang et al. 2016; Ullah et al. 2015; Fahy et al. 2018). Bone marrow MSCs are the most common source of MSCs and can promptly boost the cartilage regeneration (Richardson 2016; Gugjoo et al. 2016). Nevertheless, the process to collect bone marrow MSCs is invasive and may cause potential infections to the donors (Lukomska 2019). Human umbilical-cord-derived MSCs (hUCMSCs) are another attractive source that could be potentially used for OA treatment and they are easy to obtain and store (Ding et al. 2015; Wang 2018). However, the mechanisms underlying the chondrocyte differentiation of hUC-MSCs remains largely unknown.

Long non-coding RNAs (lncRNAs) are a class of noncoding RNAs that have been reported to play a critical role in a number of processes including physiological processes and pathological processes (Yao et al. 2019; DiStefano 2018). Changes in the expression of lncRNAs have been linked to many diseases, including cancers and OA (DiStefano 2018; Cen et al. 2017). For example, lncRNA MSR was reported to increase during OA while lncRNA H19 was diminished (Liu 2016; Steck 2012). Moreover, manipulating the expression levels of those lncRNAs could affect OA progression (Cen et al. 2017; Chen et al. 2018). Recently, lncRNA CIR was observed elevated during OA and inhibition of lncRNA CIR mitigated the progression of OA (Lu et al. 2018). However, the underlying mechanisms of how lncRNA CIR regulates chondrogenic differentiation are unclear.

Atonal homolog 8 (ATOH8), also known as Math6, is a transcription factor of the basic helix-loop-helix (bHLH) protein family (Inoue 2001). It is broadly expressed in many tissues such as the kidney, brain, and skeletal muscles, which plays a crucial role in the regulation of cell proliferation and differentiation (Chen 2011). Previous studies have indicated that $\mathrm{ATOH} 8$ regulates chondrocyte proliferation and differentiation via Indian hedgehog (Ihh) signaling (Schroeder et al. 2019). Further, reduced expression of transcription factors including ATOH8 have been observed in OA and these reductions are associated with enhanced epigenetic modifications (Alvarez-Garcia 2016). Interestingly, EZH2, a histone methyltransferase, has been implicated to repress ATOH8 expression via elevating H3K27 trimethlylation (H3K27me3) in hepatocellular carcinoma (Zhang 2019). Our initial RNA-protein interaction prediction (RPIseq) analysis suggests that lncRNA CIR might bind to EZH2. We thus hypothesized that lncRNA CIR might regulate chondrogenic differentiation through EZH2/ATOH8 pathway.

In the present study, we carried out a series of experiments (the flow chat showed in Additional file 1: Fig. S1) to investigate the function of IncRNA CIR in chondrogenic differentiation, with particular focuses on the regulation of EZH2/ATOH8 pathway. We found that lncRNA CIR was reduced while ATOH8 was increased during chondrogenic differentiation of hUC-MSCs. Both knockdown lncRNA CIR and ATOH8 overexpression facilitated chondrogenic differentiation. Further, lncRNA CIR could suppress ATOH8 expression by promoting EHZmediated H3K27 trimethylation, and silencing ATOH8 reversed the effects of lncRNA CIR knockdown on chondrogenic differentiation. Our study reveals an essential role of lncRNA CIR in chondrogenic differentiation and sheds light on the roles of hUC-MSCs in OA. These findings could provide some avenues for further OA therapeutic treatments.

\section{Materials and methods}

\section{Human umbilical-cord-derived mesenchymal stem cells} (hUC-MSCs) culture

Umbilical cords for hUC-MSCs preparation were obtained from healthy pregnant patients (24-28 years old) without infectious diseases in the Second Xiangya Hospital, Central South University (Changsha, China). The study was reviewed and approved by the Ethics Committee of the Second Xiangya Hospital, Central South University and a written informed consent was signed by each patient. Umbilical cord samples were cut into $1-2 \mathrm{~mm}^{3}$ pieces and then digested with $0.075 \%$ collagenase II (Sigma, USA) and 0.125\% trypsin (Gibco, USA) at $37^{\circ} \mathrm{C}$ for $45 \mathrm{~min}$ followed by a pass through the $100-\mu \mathrm{m}$ filter. Cell suspensions were washed with PBS and then cultured in Dulbecco's modified Eagle medium (DMEM)/F12 (1:1) (Thermo Fisher Scientific, USA) supplemented with $10 \%$ fetal bovine serum (FBS, Neuromics, USA), $1 \%$ penicillin-streptomycin (Gibco), $2 \mathrm{mM}$ L-glutamine (Gibco), and $10 \mathrm{ng} / \mathrm{mL}$ epidermal growth factor (EGF; Sigma). The cells were maintained at $37{ }^{\circ} \mathrm{C}$ 
in a humidified atmosphere containing $5 \% \mathrm{CO}_{2}$ and the medium was changed twice a week.

\section{hUC-MSC characterization}

The phenotypes of hUC-MSCs were determined by flow cytometry. Briefly, the cells were detached with $0.05 \%$ trypsin-EDTA and washed with PBS twice when they grew up to $80-90 \%$ confluence. Cell suspensions were blocked with $1 \%$ goat serum for $30 \mathrm{~min}$ at room temperature followed by incubation with different fluorescentlabeled antibodies against CD34, CD44, CD45, and CD90 (1:500; BD Biosciences, USA) at $4{ }^{\circ} \mathrm{C}$ for $30 \mathrm{~min}$. Cells were further analyzed using a FACS Calibur flow cytometer (Becton Dickinson).

\section{hUC-MSC differentiation in vitro}

For adipogenic differentiation, cells were seeded at a density of $1 \times 10^{5}$ cells/well in 6-well plates and grow up to $80 \%$ confluence. The medium was switched to adipogenic medium (DMEM supplemented with 10\% FBS) containing $10 \mu \mathrm{M}$ insulin, $0.5 \mu \mathrm{M}$ isobutylmethylxanthine, $1 \mu \mathrm{M}$ dexamethasone, and $60 \mu \mathrm{M}$ indomethacin (Sigma) for 21 days with medium changed every 3 days. Oil red oranse (Sigma) staining was performed to analyzed the differentiated adipocytes.

For osteogenic differentiation, cells were seeded at a density of $1 \times 10^{5}$ cells/well in 6-well plates and grow up to $80 \%$ confluence. The medium was switched to osteogenic medium (DMEM supplemented with 10\% FBS) containing $10 \mathrm{mM} \beta$-glycerol phosphate, $50 \mu \mathrm{M}$ ascorbate, $0.1 \mu \mathrm{M}$ dexamethasone (Sigma) for 3 weeks. Alizarin red (Sigma) staining was performed to analyze differentiated osteocytes.

For chondrogenic differentiation, cells were seeded at a density of $1 \times 10^{5}$ cells/well in 6-well plates and grow up to $80 \%$ confluence. The medium was switched to chondrogenic medium (high glucose DMEM supplemented with $10 \%$ FBS) containing $100 \mathrm{nM}$ dexamethasone, $10 \mathrm{ng} / \mathrm{ml}$ TGF- $\beta 3,50 \mathrm{ng} / \mathrm{ml}$ ascorbate-2-phosphate, and $1 \mathrm{mM}$ sodium pyruvate (Sigma) for 21 days. Alcian blue (Sigma) staining was performed to analyze differentiated chondrocytes.

\section{Plasmid and cell transfection}

si-CIR/pcDNA3.1-CIR, si-EZH2/pcDNA3.1-EZH2, si-ATOH8/pcDNA3.1-ATOH8 were purchased from GenePharma, Shanghai, China. Transfection was performed using Lipofectamine 3000 (Invitrogen, Missouri, USA) according to manufacturer's instruction. Briefly, cells $\left(1 \times 10^{5}\right.$ cells/well $)$ were cultured in 6 -well plates and grown until $80 \%$ confluence. $1 \mu \mathrm{g}$ of plasmid was used for transfection together with $1 \mu \mathrm{L}$ Lipofectamine 3000 .
Cells were subjected to differentiation immediately following transfection and then harvested at indicated time.

\section{Subcellular fractionation}

Subcellular fractionation was performed as previously described (Tan et al. 2015, 2020). Briefly, the cells were homogenerated in homogeneration buffer $[320 \mathrm{mM}$ sucrose, $5 \mathrm{mM}$ sodium pyrophosphate, $1 \mathrm{mM}$ EDTA, $10 \mathrm{mM}$ Hepes ( $\mathrm{pH}$ 7.4)] supplemented with protease inhibitor (Roche). Homogenate was then centrifuged at $800 \times g$ for $10 \mathrm{~min}$ at $4{ }^{\circ} \mathrm{C}$ to yield nuclei (pellet). Supernatant was collected and centrifuged at $20,000 \times g$ for $20 \mathrm{~min}$ to yield membrane fraction (pellet) and cytoplasm fraction (supernatant).

\section{RNA extraction and qRT-PCR}

Total RNA was isolated from treated cells using Trizol reagent (Invitrogen) according to the manufacturer's instructions. DNaseI was added into the lysis buffer to avoid DNA contamination. Cytoplasmic and Nuclear RNA purification was perfumed using the purification kit (Norgen, Canada) according to the manufacturer's protocol. $1 \mu \mathrm{g}$ total RNA from each sample was subjected to reverse transcription using the SuperScript ${ }^{\circledR}$ IV First-Strand Synthesis System (Invitrogen) before PCR amplification using $1 \times$ Power SYBR ${ }^{\circledR}$ Green PCR Master Mix (Invitrogen). Relative expression levels of lncRNA or mRNA were normalized to 18S RNA and GAPDH mRNA, respectively, as internal controls. The following primers were used in this study:

lncRNA CIR forward primer: 5'-ACACTTGCAAGC CTGGGTAG- $3^{\prime}$

lncRNA CIR reverse primer: 5'-CCATTTTCCTGT TGGTGCGG-3'

ATOH8 forward primer: 5'-CCTGAGGATCGCCTG TAACT-3'

ATOH8 reverse primer: 5'-TGTCAAAGGCTCCGA AAAGT- $3^{\prime}$

18S RNA forward primer: 5'- CAGGATTGACAGATT GATAGC TCT- $3^{\prime}$

18S RNA reverse primer: $5^{\prime}$-GAGTCTCGTTCGT TAT CGGAATTA-3'

GAPDH forward primer: 5'-CCAGGTGGTCTCCTC TGA-3'

GAPDH reverse primer: 5'-GCTGTAGCCAAATCG TTGT-3'

\section{Western blotting}

Total protein was extracted from treated cells with RIPA lysis buffer (Beyotime Institute of Biotechnology, Nantong, China) and the concentration of the protein 
was determined with Pierce ${ }^{\mathrm{TM}}$ BCA Protein Assay Kit (Thermo Fisher Scientific). Protein samples were separated by $12 \%$ SDS-polyacrylamide gels and then transferred to PVDF membranes (EMD Millipore, USA). The membranes were blocked for $1 \mathrm{~h}$ at room temperature. Subsequently, primary antibodies were added and incubated overnight at $4{ }^{\circ} \mathrm{C}$. Following washes with TBST, the membranes were incubated with a secondary antibody for $1 \mathrm{~h}$ at room temperature. Protein bands were visualized using the ECL kit. The following primary antibodies were used: Anti-ATOH8 (1:1000; Abcam, USA); Antisex determining region Y-box 9 (SOX9) (1:1000; Abcam); Anti-Aggrecan (1:500; Abcam); Anti-type-II collagen (Col2A1) (1:500; Santa Cruz, USA); Anti-EZH2 (1:1000; Cell Signaling Technology, USA); Anti-H3K27me3 (1:1000; Abcam); Anti-GAPDH (1:10,000; Abcam); Anti$\beta$-actin (1:1000; Santa Cruz).

\section{RNA Immunoprecipitation (RIP) assay}

Transfected cells were lysed in lysis buffer $(50 \mathrm{mM}$ Tris-HCl, $150 \mathrm{mM} \mathrm{NaCl}, 2 \mathrm{mM}$ EDTA, 1\% NP-40, 0.5\% sodium deoxycholate) containing RNase inhibitors and protease inhibitors (Thermo Scientific, Waltham, MA, USA). The extracted protein was incubated with relevant antibodies (anti-EZH2, 1:1 000 dilution and IgG as control) (Abcam) overnight at $4{ }^{\circ} \mathrm{C}$ and then pulled down with protein $\mathrm{G}$ Sepharose 4 Fast Flow suspension (GE Amersham, Little Chalfont, UK). The beads were digested with proteinase K (Sangon, Shanghai, China) for $1 \mathrm{~h}$ followed by RNA purification with Trizol reagent (Invitrogen). Quantitative RT-PCR was performed to examine the RNA yield.

\section{Biotin pull-down assay}

The biotinylated IncRNA CIR was prepared using the MEGshortscript ${ }^{\mathrm{TM}}$ T7 kit based on the manufacturer's protocol and then incubated with cell lysates from hUCMSCs at $4{ }^{\circ} \mathrm{C}$ for $1 \mathrm{~h}$ followed by mixture with streptavidin-coupled dynabeads (Invitrogen, Shanghai, China) at $4{ }^{\circ} \mathrm{C}$ for another $3 \mathrm{~h}$. The beads were then washed with TENT (10 mM Tris-HCl [pH 8.0], 1 mM EDTA [pH 8.0], $250 \mathrm{mM} \mathrm{NaCl}, 0.5 \%$ Triton X-100) buffer 3 times and eluted with $1 \times$ laemmli SDS sample buffer. The elution was heated for $5 \mathrm{~min}$ at $95{ }^{\circ} \mathrm{C}$ and proceeded to western blotting analysis of EZH2 and H3K27me3.

\section{Chromatin immunoprecipitation (ChIP) assay}

ChIP was performed using the commercial ChIP kit (Cell Signaling Technology) according to the manufacturer's protocol. For each chromatin immunoprecipitation, $5 \mu \mathrm{g}$ of anti-EZH2 or anti-H3K27me3 antibodies and $1 \mu \mathrm{L}$ of normal IgG were used. After immunoprecipitation, chromosomal DNA was purified. ATOH8 promoter region was detected by using PCR. The primers used for amplifying the ATOH8 promoter region were as follows: forward: 5'-GCGTGACTTTGGAGCTTTCG-3'; reverse: $5^{\prime}$-ACTCGCCACGAGACAGAAAA- ${ }^{\prime}$. The results were expressed as relative input.

\section{Immunostaining}

Cells were fixed in $4 \%$ paraformaldehyde (PFA) at room temperature for $15 \mathrm{~min}$ and permeabilized with $0.1 \%$ Triton X-100 in PBS for half an hour at room temperature. Then cells were blocked with $1 \%$ BSA in PBS for $1 \mathrm{~h}$ at room temperature followed by incubation with primary antibodies at $4{ }^{\circ} \mathrm{C}$ overnight. Cells were then washed with PBS and incubated with secondary antibody conjugated with TRITC conjugated secondary antibody for $2 \mathrm{~h}$ at room temperature. Images were acquired with standard microscope.

\section{Statistical analysis}

All cell experiments were performed at least three times and analyzed using Graphpad Prism 8. Statistical significance was determined by unpaired two-tailed Student's $t$ test for two groups or one-way ANOVA followed by Tukey's post hoc test for multiple groups. ${ }^{*} P<0.05$, ${ }^{* *} P<0.01$, ${ }^{* * *} P<0.001$. Data were presented as Mean \pm SD (standard deviation).

\section{Results}

Isolation and identification of hUC-MSCs

We first characterized the identities of hUC-MSCs. Human umbilical cord tissues were cultured for more than 2 weeks and spindle-shaped fibroblastic cells were observed Fig. 1b. MSCs are known to express CD13, CD29, CD44, CD90, CD105, but not CD34, CD45, CD117 (Ullah et al. 2015). Flow cytometry analysis revealed that our isolated hUC-MSCs were positive for CD44 and CD90 Fig. 1a, but negative for CD34 and CD45 signals, indicating that the cultured cells exhibited MSC features.

To characterize the differentiation potentials of hUCMSCs, we cultured hUC-MSCs in various media including adipogenic, osteogenic, and chondrogenic medium for 3 weeks and then analyzed their differentiation abilities using correlated staining methods. Cells growing in osteogenic medium showed positive Alizarin red staining Fig. 1c, cells cultured in adipogenic medium exhibited positive oil red oranse staining Fig. 1d, and 


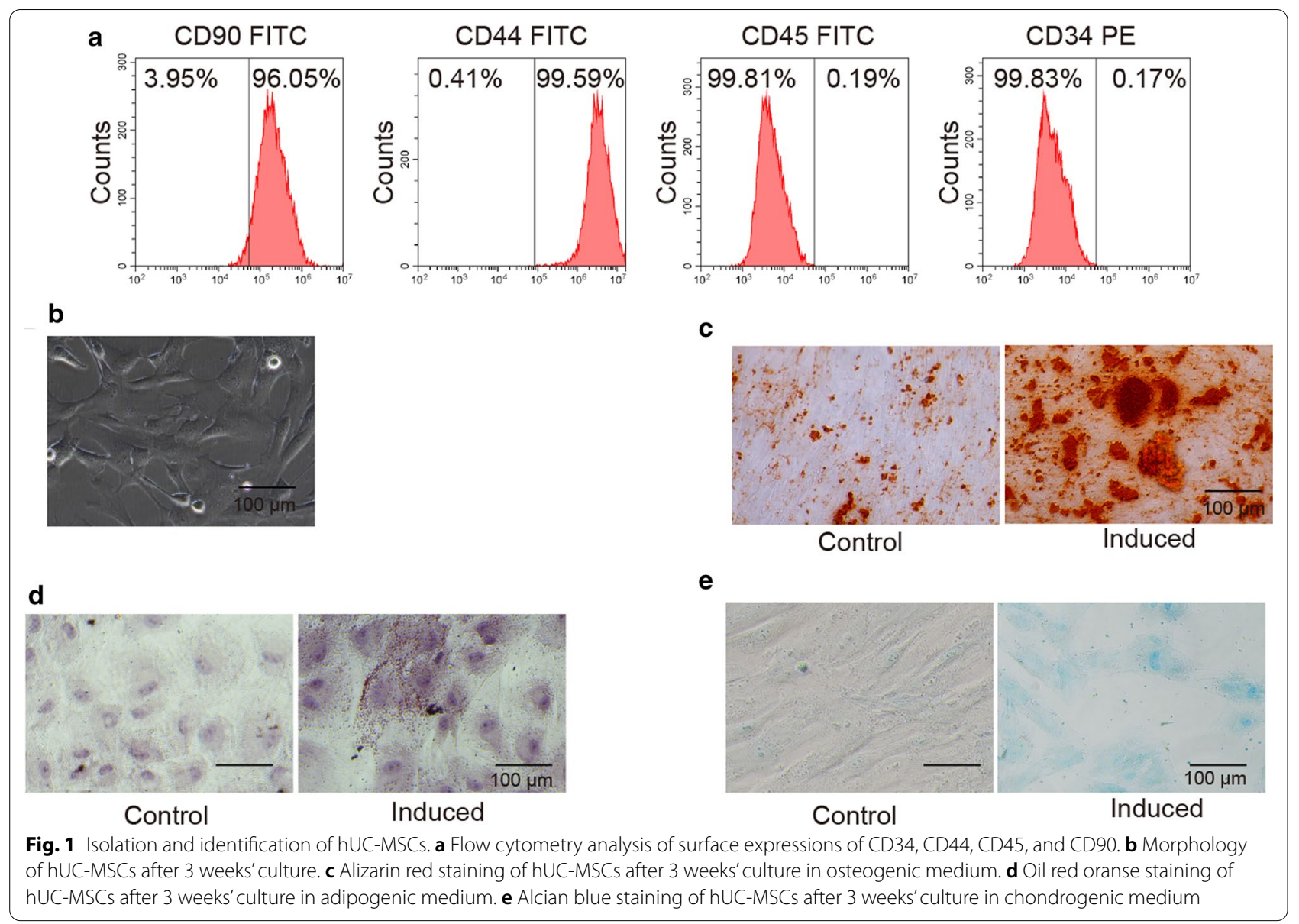

cells in chondrogenic medium revealed Alcian blue signal Fig. 1e. Together, these results demonstrate that our hUC-MSCs could differentiate into chondrocytes under specific conditions.

\section{LncRNA CIR knockdown promotes chondrogenic differentiation of hUC-MSCs}

To examine the role of lncRNA CIR in chondrogenic differentiation, we first measured the level of lncRNA CIR during chondrogenic differentiation. With hUCMSCs, we observed that lncRNA CIR was significantly reduced Fig. 2a, while both mRNA level and protein level of ATOH8 were elevated during chondrogenic differentiation Fig. 2b, c. Next, we manipulated lncRNA CIR level through siRNA and examined how it affected the chondrogenic differentiation. Cells transfected with si-CIR had much lower level of lncRNA CIR compared with control cells (non-transfected cells) or cells transfected with si-NC Fig. 2d. Interestingly, there were more Alcian blue positive cells when lncRNA CIR was knocked down through si-CIR compared with control cells, although the change was not very strong Fig. 2e. Moreover, hUC-MSCs transfected with si-CIR had higher levels of chondrogenic markers, such as SOX9, Aggrecan, Col2A1 than non-transfected cells or cells transfected with si-NC Fig. 2f. Immunostaining method also revealed higher signals of Col2A1 and Aggrecan in si-CIR treated cells Fig. 2g. Therefore, we conclude that lncRNA CIR knockdown could promote chondrogenic differentiation of hUC-MSCs evidenced by increase of chondrogenic marker expression.

\section{Overexpression of ATOH8 promotes chondrogenic differentiation of hUC-MSCs}

We then investigated how ATOH8 regulated chondrogenic differentiation. Ectopic expression of pcDNA3.1ATOH8 resulted in higher levels of ATOH8 mRNA, as well as ATOH8 protein Fig. 3a, b. We found that, similar to lncRNA CIR knockdown, increasing ATOH8 level had a small tendency to up-regulate the number of Alcian blue positive cells Fig. 3c. In addition, expressions of chondrogenic markers including SOX9, 

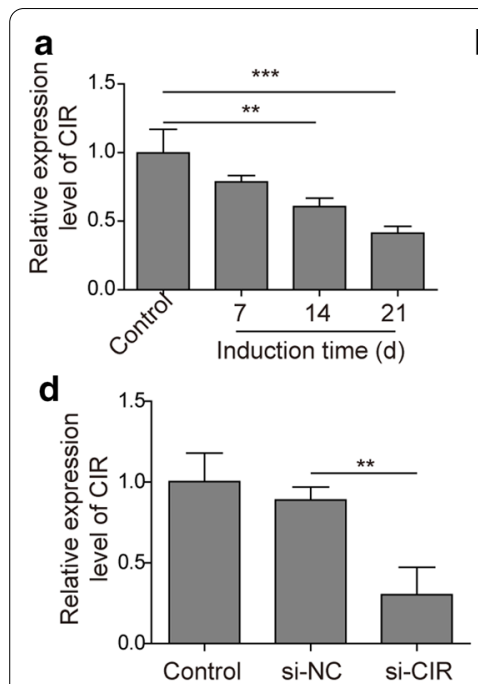

f

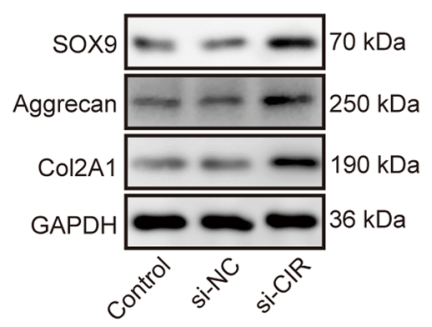

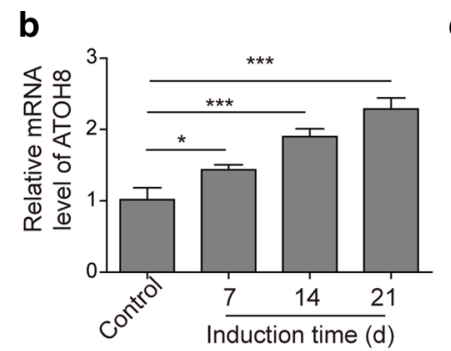
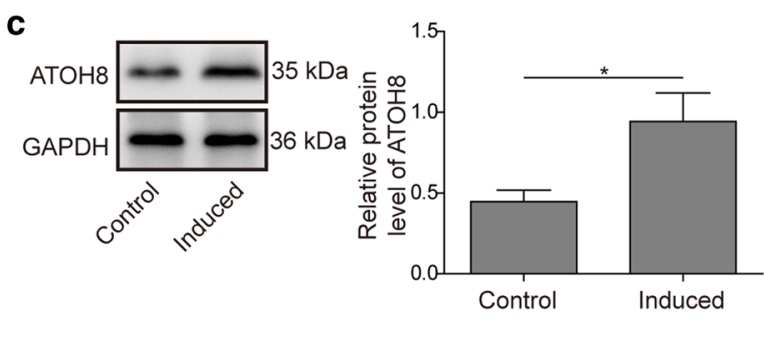

e
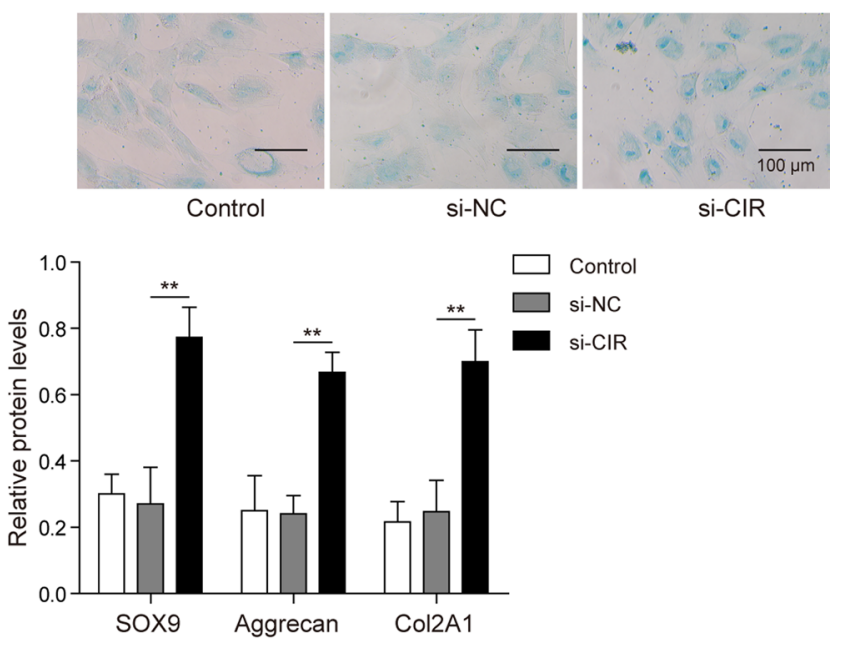

g
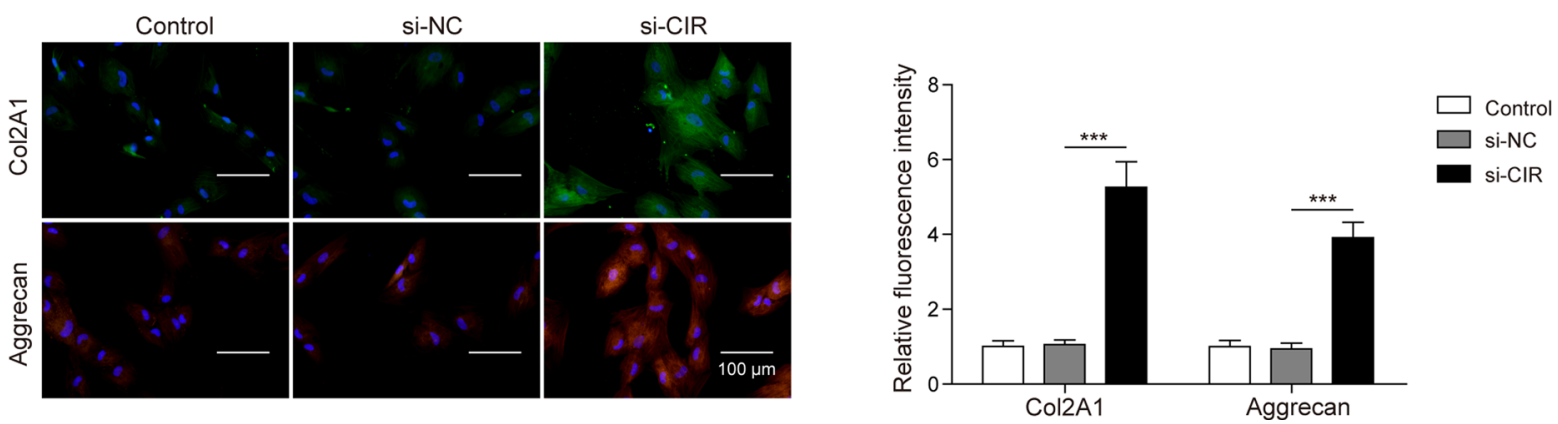

Fig. 2 LnCRNA CIR knockdown promotes chondrogenic differentiation of hUC-MSCs. a qRT-PCR analysis of IncRNA CIR level in control and differentiated hUC-MSCs. b qRT-PCR analysis of ATOH8 mRNA level in control and differentiated hUC-MSCs. c Western blot analysis of ATOH8 protein level in control and differentiated hUC-MSCs. d qRT-PCR analysis of IncRNA CIR level in different transfected hUC-MSCs. e Alcian blue staining to analyze chondrogenic differentiation of hUC-MSCs transfected with different constructs. $\mathbf{f}$ Western blot analysis of levels of chondrogenic markers including SOX9, Aggrecan, and Col2A1 in transfected cells. $\mathbf{g}$ Immunostaining analysis of levels of Col2A1 and Aggrecan in transfected cells. $\mathrm{n}=3$; ${ }^{*} P<0.05$; ${ }^{* *} P<0.01$; ${ }^{* *} P<0.001$

Aggrecan, and Col2A1 were all elevated by ATOH8 overexpression Fig. 3d, e. Taken together, these data show that ATOH8 may be a promoter in chondrogenic differentiation of hUC-MSCs.

\section{LncRNA CIR directly binds to EZH 2}

We next sought to examine the regulatory relationship between lncRNA CIR and ATOH8, since they both regulated chondrogenic differentiation. EZH2, a histone methyltransferase, is the functional enzymatic component of the Polycomb Repressive Complex 2 (PRC2) (Tan et al. 2014). Changes of EZH2 directly affect the thrimethylation of H3K27 (H3K27me3) and methlylation of CpG islands (Pan et al. 2018), leading to alternations of many proteins, including ATOH8 (Zhang 2019). With some preliminary RPIseq analysis, we found that lncRNA 


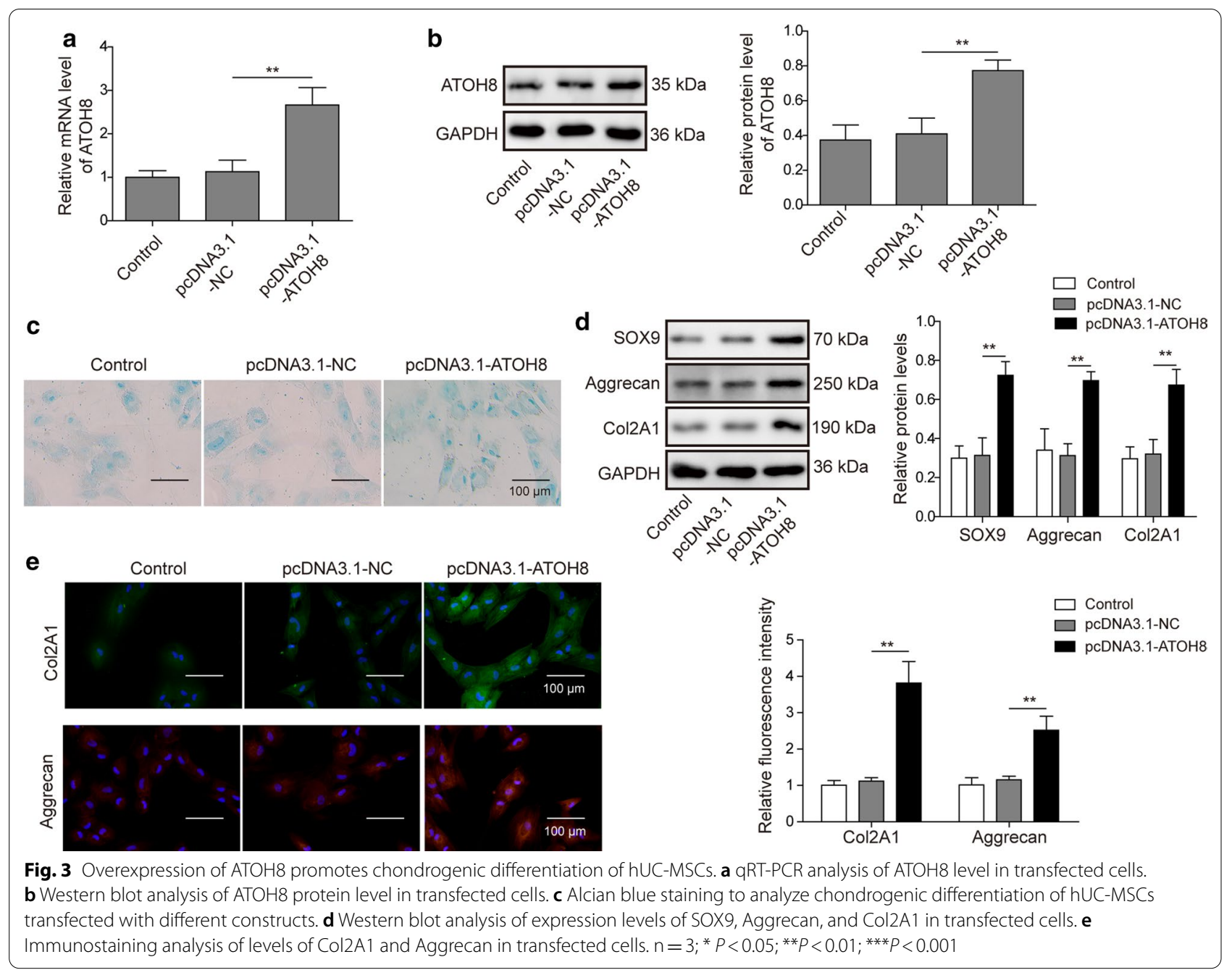

CIR might bind to EZH2 (data not shown). To confirm this prediction, we first looked at subcellular localization of lncRNA CIR as EZH2 is predominantly localized to nucleus. With subcellular fractionation, as expected, we found that GAPDH was mainly localized in cytoplasm while U6 was predominantly expressed in nucleus
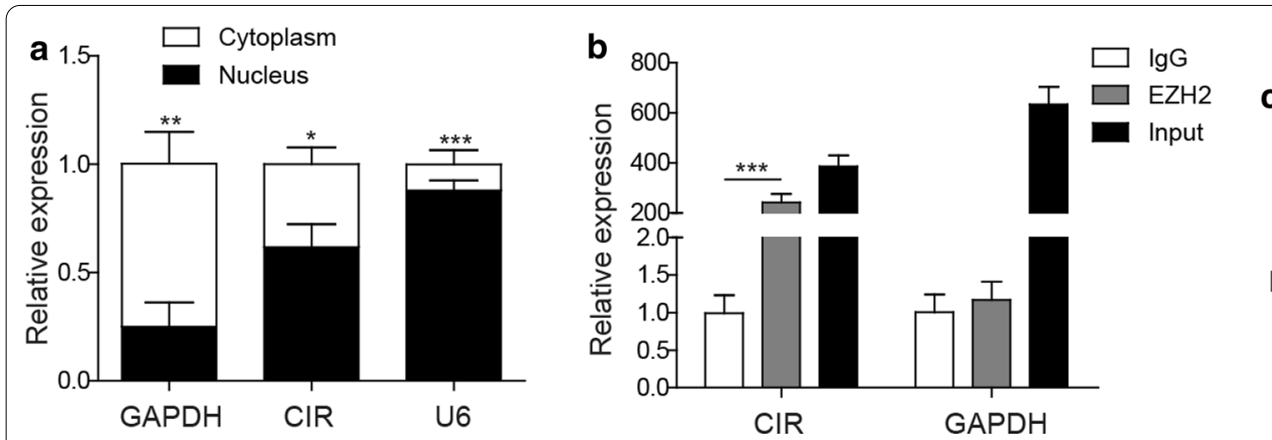

C

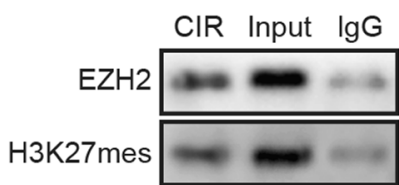

Fig. 4 LncRNA CIR directly binds to EZH2. a qRT-PCR analysis of IncRNA CIR level in cytoplasm and nucleus. $\mathbf{b}$ RIP assay was performed to analyze InCRNA CIR/EZH2 interaction. $\mathbf{c}$ Biotin-RNA pull down assay was used to measure the interactions of IncRNA CIR with EZH2 and $\mathrm{H} 3 \mathrm{~K} 27 \mathrm{me}$. $\mathrm{n}=3$; ${ }^{*} P<0.05 ;{ }^{*} P<0.01 ;{ }^{* *} P<0.001$ 
Fig. 4a, indicating that we successfully isolated cytoplasm fraction and nucleus fraction. Regarding lncRNA CIR, we showed that the majority of IncRNA CIR was in the nucleus Fig. 4a, which is similar to EZH2. Using RIP assay, we found that immunoprecipitation with EZH2 antibody successfully pulled down lncRNA CIR Fig. 4b, indicating that lncRNA CIR binds to EZH2. Recent studies suggest that EZH2 represses ATOH8 expression via regulating methylation of its promoter and H3K27 trimethylation (Zhang 2019). We next performed RNA precipitation with a biotinylated lncRNA CIR probe and the results showed specific enrichments of EZH2 and H3K27me3 in the precipitates of lncRNA CIR Fig. 4c, indicating that lncRNA CIR binds to EZH2 and H3K27me3.

\section{LncRNA CIR binds to EZH2 and silences ATOH8 expression}

The results presented above suggest that lncRNA CIR might regulate ATOH8 expression via binding to EZH2. We next directly examined that regulation. Knockdown EZH2 through si-EZH2 significantly diminished the levels of EZH2 and H3K27me3 but upregulated the level of ATOH8, while EZH2 overexpression showed the opposite trends Fig. 5a. The ATOH8 promoter is located in a typical CpG island and EZH2 suppresses ATOH8 expression through raising methylation of its promoter (Zhang 2019). Through ChIP-qRT-PCR, we found that silencing
lncRNA CIR significantly weakened the bindings of EZH2 and H3K27me3 to ATOH8 promoter Fig. 5b, suggesting that lncRNA CIR promotes ATOH8 methylation. Further, EZH2 knockdown upregulated ATOH8 expression and downregulated H3K27me3 level, but overexpression of lncRNA CIR reversed the effects of EZH2 knockdown Fig. 5c, d. All together, these results demonstrate that lncRNA CIR could repress ATOH8 expression via promoting EZH2-mediated methylation.

\section{LncRNA CIR inhibits chondrogenic differentiation by epigenetically inhibiting ATOH 8}

In the end, we sought to reveal the mechanisms of how lncRNA CIR regulates chondrogenic differentiation of hUC-MSCs. Consist with aforementioned results, knockdown lncRNA CIR in hUC-MSCs increased ATOH8 level while overexpression of IncRNA CIR decreased Fig. 6 a. Silencing ATOH8 mRNA through si-ATOH8 greatly reduced ATOH8 protein level as well Fig. 6b. Consistently, knockdown lncRNA CIR facilitated Alcian blue positive cells and silencing ATOH8 suppressed that, but these differences were not clear Fig. 6c. However, cells transfected with si-CIR had higher expressions of chondrogenic markers including SOX9, Aggrecan and Col2A1 while cells transfected with si-ATOH8 had lower levels

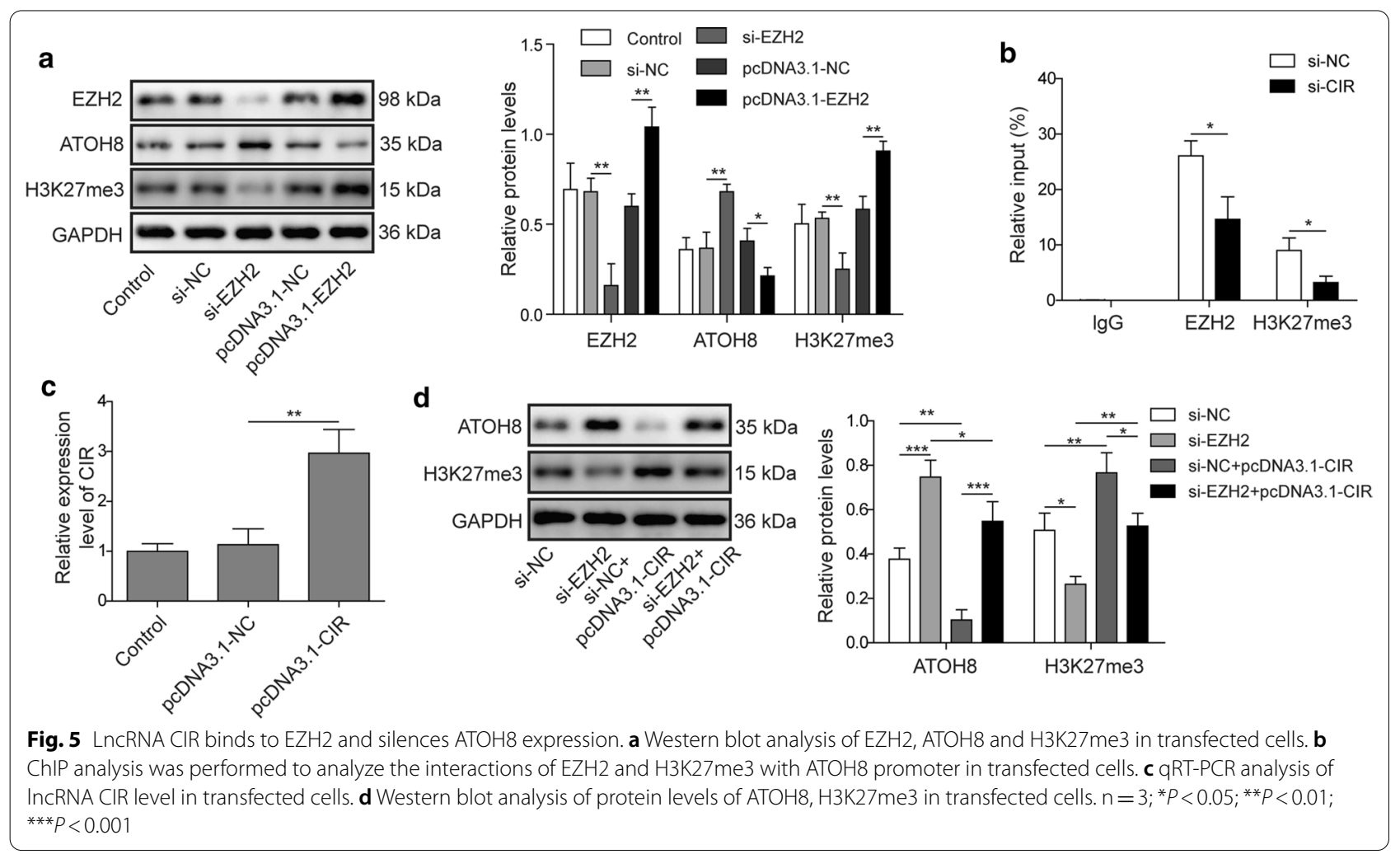




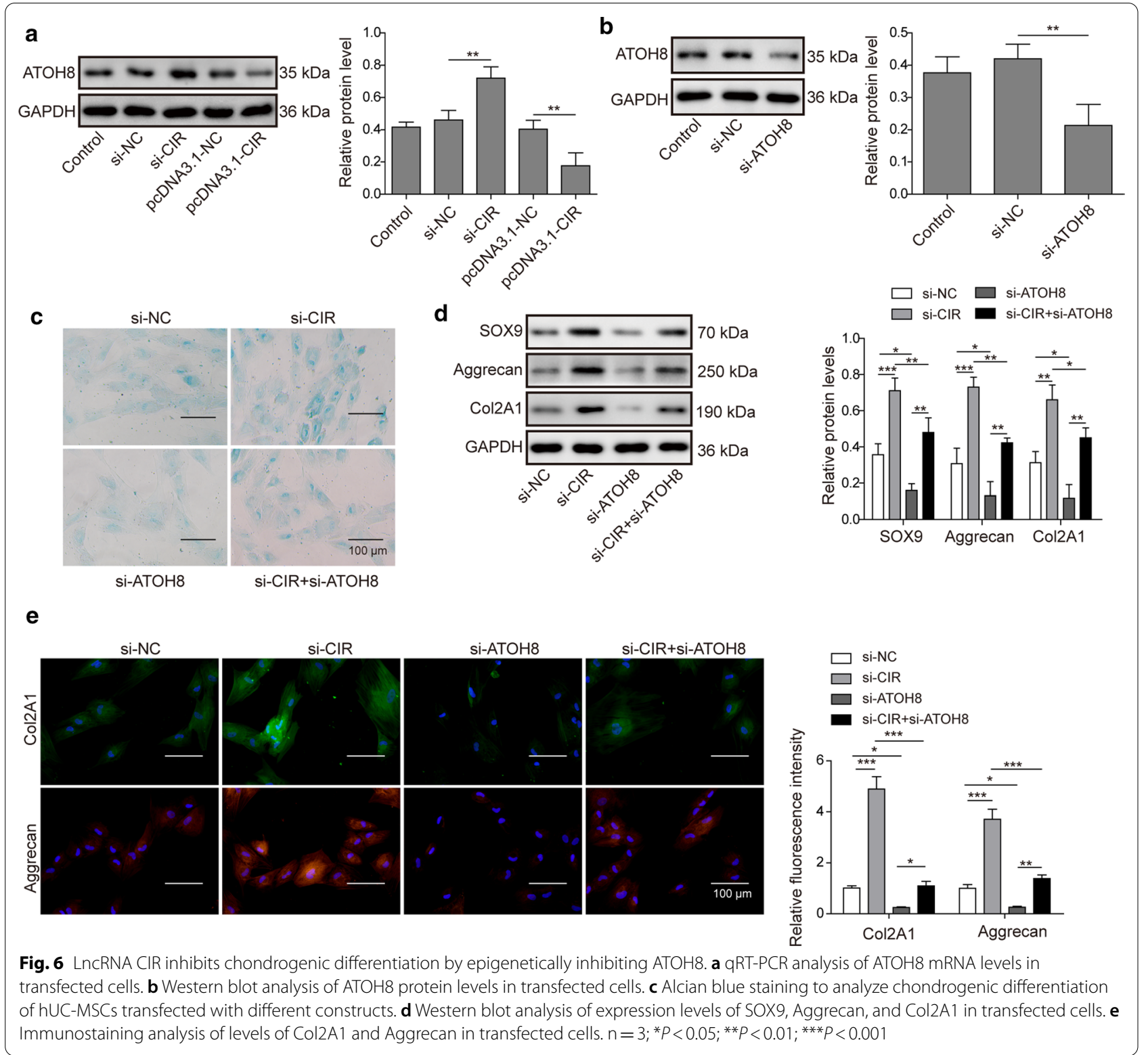

compared to control cells Fig. 6d, e. Moreover, si-ATOH8 inhibited those upregulations mediated by lncRNA CIR knockdown Fig. 6d, e, suggesting that lncRNA CIR may suppress chrondrogenic differentiation through downregulation of ATOH8.

\section{Discussion}

$\mathrm{OA}$ is a chronic disorder caused by degeneration of the joint cartilage, leading to joint malfunction, pain, and disability (Vina and Kwoh 2018; Martel-Pelletier 2016). The prevalence is very high and the incidence of $\mathrm{OA}$ is progressively increasing, as it is highly associated with age (Vina and Kwoh 2018). However, the underlying mechanisms of OA development and progression are elusive. In the present study, we investigated the function of lncRNA CIR in OA with particular focus on the chondrogenic differentiation of hUC-MSCs. We observed that IncRNA CIR was decreased but ATOH8 was increased during chondrogenic differentiation. Knockdown lncRNA CIR may increase ATOH8 expression via repressing EZH2-mediated methylation and promoted chondrogenic differentiation. These results demonstrated an essential role of lncRNA CIR/EZH2/ATOH8 in chondrogenic differentiation, which could serve as a potential target for OA therapeutic development. 
Chondrocytes are the only cells in the cartilage and are responsible for maintaining tissue homeostasis in the cartilage (Akkiraju and Nohe 2015). OA is characterized by degenerative chondrocytes (Singh et al. 2019). Previous studies have identified a huge number of hypermethylated transcription factors including ATOH8 in OA wherein the functions of chondrocytes are compromised, resulting in reduced expressions (Alvarez-Garcia 2016). In addition, lncRNA CIR has been shown elevated in OA cartilage (Lu et al. 2018). Here, we observed opposite changes of ATOH8 and lncRNA CIR during chondrogenic differentiation of hUC-MSCs. These results are very consistent and together with other studies (Schroeder et al. 2019; Alvarez-Garcia 2016; Wang et al. 2018; Liu 2014), supporting the model that lncRNA CIR inhibits chondrogenic differentiation while ATOH8 facilitates chondrogenic differentiation. Therefore, restoring the expression of ATOH8 or inhibition of lncRNA CIR could promote the differentiation of chondrocytes evidenced by expression of chondrogenic markers including SOX9, Aggrecan and Col2A1 and retard the development or progression of OA. Notably, accumulating evidence indicates that non coding RNAs play crucial roles in the OA, including lncRNAs and miRNAs, and that they could serve as therapeutic targets (Sun 2019). For instance, suppressing lncRNA HOTAIR could inhibit the cartilage degradation during OA (Yang 2020). It will be interesting to study the functions of other non-coding RNAs in OA.

LncRNAs are long non-coding RNAs that play important roles in regulating gene expressions during various cellular processes (Yao et al. 2019). Many lncRNAs have been reported to function as sponges of miRNA, which results in dis-inhibition of target gene expression (Zhang et al. 2018, 2016; Yue 2019). Here, we found lncRNA CIR regulated ATOH8 expression via a distinct mechanism. lncRNA CIR could bind to EZH2 and promotes EZH2-mediated H3K27 trimethylation of ATOH8, leading to reduced expression of ATOH8 protein. This finding suggests that lncRNA can modulate gene expressions through diverse mechanisms. Indeed, many other studies have demonstrated that IncRNAs directly interact with proteins involved in the regulation of chromatin conformation, indicating the interplay between IncRNA and epigenetic machinery (Neve et al. 2018; Morlando and Fatica 2018; Wang 2017; Han and Chang 2015). There may be other mechanisms underlying the effects of lncRNA CIR on ATOH8 expression and it might be interesting for future study.

hUC-MSCs are the very attractive avenue for regenerative medicine compared to bone marrow MSCs (Ding et al. 2015; Detamore 2013). Consistent with previous studies, we demonstrated that hUC-MSCs have great differentiation potential and can be differentiated into various types of cells with specific conditions, including chondrocytes (Wang 2018; Zhao 2019). Moreover, our work reveals a key role of lncRNA CIR/EZH2/ATOH8 signaling in chondrogenic differentiation process. Since our study is limited by in vitro cell culture system, it is very necessary to confirm these results with in vivo animal models. Additionally, three-dimensional (3D) cell culture system is a very useful in vitro cell model for

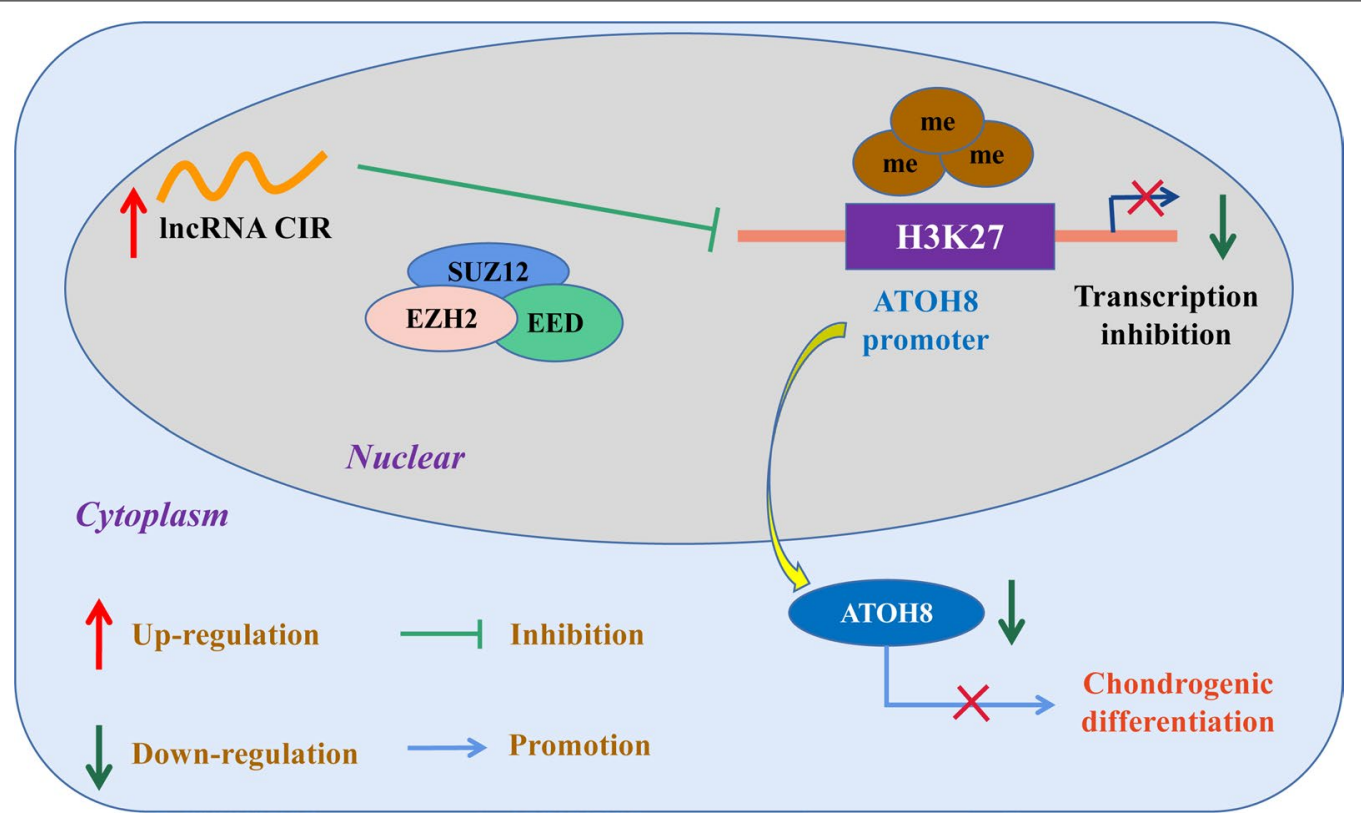

Fig. 7 Schematic diagram demonstrates the role of IncRNA CIR/EZH2/ATOH8 axis in chondrogenic differentiation 
diseases (Langhans 2018). It will be interesting to further study the role of lncRNA CIR/EZH2/ATOH8 signaling in chondrogenic differentiation with 3D cell model.

In summary, our work presented here demonstrates that lncRNA CIR may regulate chondrogenic differentiation of hUC-MSCs via EZH2/ATOH8 pathway Fig. 7. Targeting lncRNA CIR/EZH2/ATOH8 pathway could be a potential avenue for future $\mathrm{OA}$ therapeutic development.

\section{Supplementary Information}

The online version contains supplementary material available at https://doi. org/10.1186/s10020-021-00272-9.

Additional file 1: Figure S1. The flow chart of experimental procedure in this study.

\section{Acknowledgements}

None.

\section{Authors' contributions}

This study was designed by FL and J-DN. D-YS made great contribution to the data collection of this study, and $\mathrm{JH}$ and $\mathrm{H}$-QY were responsible for the data analysis and ascertained the integrity as well as accuracy of the data. FL and DY prepared and approved this manuscript. All authors read and approved the final manuscript.

\section{Funding}

This work was supported by the Xiangya Famous Doctors of Central South University (No.201468)

\section{Availability of data and materials}

All data generated or analyzed during this study are included in this published article.

\section{Ethics approval and consent to participate}

The study was reviewed and approved by the Ethics Committee of the Second Xiangya Hospital, Central South University and a written informed consent was signed by each patient.

\section{Consent for publication}

The informed consent obtained from study participants.

\section{Competing interests}

The authors declare that they have no competing interests.

\section{Author details}

${ }^{1}$ Department of Orthopedics, The Second Xiangya Hospital of Central South University, No.139 Renminzhong Road, Changsha 410011, Hunan, People's Republic of China. ${ }^{2}$ Department of Orthopedics, The Third Hospital of Changsha, Changsha 410015, Hunan, People's Republic of China.

Received: 9 September 2020 Accepted: 13 January 2021 Published online: 05 February 2021

\section{References}

Akkiraju H, Nohe A. Role of chondrocytes in cartilage formation, progression of osteoarthritis and cartilage regeneration. J Dev Biol. 2015;3:177-92.

Alvarez-Garcia O, et al. Increased DNA methylation and reduced expression of transcription factors in human osteoarthritis cartilage. Arthritis Rheumatol. 2016;68:1876-86.

Caldwell KL, Wang J. Cell-based articular cartilage repair: the link between development and regeneration. Osteoarthritis Cartilage. 2015:23:351-62.
Carballo CB, Nakagawa Y, Sekiya I, Rodeo SA. Basic science of articular cartilage. Clin Sports Med. 2017;36:413-25.

Cen X, Huang XQ, Sun WT, Liu Q, Liu J. Long noncoding RNAs: a new regulatory code in osteoarthritis. Am J Transl Res. 2017;9:4747-55.

Chang YH, Liu HW, Wu KC, Ding DC. Mesenchymal stem cells and their clinical applications in osteoarthritis. Cell Transplant. 2016;25:937-50.

Chen J, et al. Diversification and molecular evolution of ATOH8, a gene encoding a bHLH transcription factor. PLOS ONE. 2011;6:e23005.

Chen L, Zhang Y, Rao Z, Zhang J, Sun Y. Integrated analysis of key mRNAs and IncRNAs in osteoarthritis. Exp Ther Med. 2018;16:1841-9.

Detamore MS. Human umbilical cord mesenchymal stromal cells in regenerative medicine. Stem Cell Res Ther. 2013:4:142.

Ding DC, Chang YH, Shyu WC, Lin SZ. Human umbilical cord mesenchymal stem cells: a new era for stem cell therapy. Cell Transplant. 2015;24:339-47.

DiStefano JK. The emerging role of long noncoding RNAs in human disease. Methods Mol Biol. 2018;1706:91-110.

Fahy N, Alini M, Stoddart MJ. Mechanical stimulation of mesenchymal stem cells: implications for cartilage tissue engineering. J Orthop Res. 2018:36:52-63.

Fransen M, et al. The epidemiology of osteoarthritis in Asia. Int J Rheum Dis. 2011:14:113-21.

Gugjoo MB, Amarpal SGT, Aithal HP, Kinjavdekar P. Cartilage tissue engineering: role of mesenchymal stem cells along with growth factors \& scaffolds. Indian J Med Res. 2016;144:339-47.

Han P, Chang CP. Long non-coding RNA and chromatin remodeling. RNA Biol. 2015:12:1094-8.

Inoue $\mathrm{C}$, et al. Math6, a bHLH gene expressed in the developing nervous system, regulates neuronal versus glial differentiation. Genes Cells. 2001;6:977-86

Langhans SA. Three-dimensional in vitro cell culture models in drug discovery and drug repositioning. Front Pharmacol. 2018;9:6.

Lepetsos P, Papavassiliou AG. ROS/oxidative stress signaling in osteoarthritis. Biochim Biophys Acta. 2016;1862:576-91.

Liu Q, et al. Long noncoding RNA related to cartilage injury promotes chondrocyte extracellular matrix degradation in osteoarthritis. Arthritis Rheumatol. 2014;66:969-78.

Liu Q, et al. The TMSB4 pseudogene LncRNA functions as a competing endogenous RNA to promote cartilage degradation in human osteoarthritis. Mol Ther 2016:24:1726-33.

Lu Z, Luo M, Huang Y. IncRNA-CIR regulates cell apoptosis of chondrocytes in osteoarthritis. J Cell Biochem. 2018. https://doi.org/10.1002/ jcb.27997.

Lukomska B, et al. Challenges and controversies in human mesenchymal stem cell therapy. Stem Cells Int. 2019;2019:9628536.

Martel-Pelletier J, et al. Osteoarthritis. Nat Rev Dis Primers. 2016;2:16072.

Mehl J, Imhoff AB, Beitzel K. Osteoarthritis of the shoulder: pathogenesis, diagnostics and conservative treatment options. Orthopade. 2018:47:368-76.

Morlando M, Fatica A. Alteration of epigenetic regulation by long noncoding RNAs in cancer. Int J Mol Sci. 2018. https://doi.org/10.3390/ijms19020570.

Neve B, Jonckheere N, Vincent A, Van Seuningen I. Epigenetic regulation by IncRNAs: an overview focused on UCA1 in Colorectal cancer. Cancers (Basel). 2018. https://doi.org/10.3390/cancers10110440.

Pan MR, Hsu MC, Chen LT, Hung WC. Orchestration of H3K27 methylation: mechanisms and therapeutic implication. Cell Mol Life Sci. 2018;75:209-23.

Richardson SM, et al. Mesenchymal stem cells in regenerative medicine: focus on articular cartilage and intervertebral disc regeneration. Methods. 2016;99:69-80

Schroeder N, Wuelling M, Hoffmann D, Brand-Saberi B, Vortkamp A. Atoh8 acts as a regulator of chondrocyte proliferation and differentiation in endochondral bones. PLoS ONE. 2019;14:e0218230.

Singh P, Marcu KB, Goldring MB, Otero M. Phenotypic instability of chondrocytes in osteoarthritis: on a path to hypertrophy. Ann N Y Acad Sci. 2019:1442:17-34

Steck $\mathrm{E}$, et al. Regulation of $\mathrm{H} 19$ and its encoded microRNA-675 in osteoarthritis and under anabolic and catabolic in vitro conditions. J Mol Med (Berl). 2012;90:1185-95. 
Sun $\mathrm{H}$, et al. Emerging roles of long noncoding RNA in chondrogenesis, osteogenesis, and osteoarthritis. Am J Transl Res. 2019;11:16-30.

Tan JZ, Yan Y, Wang XX, Jiang Y, Xu HE. EZH2: biology, disease, and structurebased drug discovery. Acta Pharmacol Sin. 2014;35:161-74.

Tan HL, Queenan BN, Huganir RL. GRIP1 is required for homeostatic regulation of AMPAR trafficking. Proc Natl Acad Sci U S A. 2015;112:10026-31.

Tan HL, Chiu SL, Zhu Q, Huganir RL. GRIP1 regulates synaptic plasticity and learning and memory. Proc Natl Acad Sci U S A. 2020;117:25085-91.

Ullah I, Subbarao RB, Rho GJ. Human mesenchymal stem cells - current trends and future prospective. Biosci Rep. 2015. https://doi.org/10.1042/BSR20 150025 .

Vargas Negrin F, Medina Abellan MD, Hermosa Hernan JC, de Felipe MR. Treatment of patients with osteoarthritis. Aten Primaria. 2014;46(Suppl 1):39-61.

Vina ER, Kwoh CK. Epidemiology of osteoarthritis: literature update. Curr Opin Rheumatol. 2018;30:160-7.

Wang C, et al. LncRNA structural characteristics in epigenetic regulation. Int J Mol Sci. 2017. https://doi.org/10.3390/ijms18122659.

Wang CL, Peng JP, Chen XD. LncRNA-CIR promotes articular cartilage degeneration in osteoarthritis by regulating autophagy. Biochem Biophys Res Commun. 2018;505:692-8.

Wang $\mathrm{H}$, et al. The human umbilical cord stem cells improve the viability of OA degenerated chondrocytes. Mol Med Rep. 2018;17:4474-82.

Yang $\mathrm{Y}$, et al. A long non-coding RNA, HOTAIR, promotes cartilage degradation in osteoarthritis by inhibiting WIF-1 expression and activating Wnt pathway. BMC Mol Cell Biol. 2020;21:53.
Yao RW, Wang Y, Chen LL. Cellular functions of long noncoding RNAs. Nat Cell Biol. 2019;21:542-51.

Yue B, et al. Characterization of IncRNA-miRNA-mRNA network to reveal potential functional ceRNAs in bovine skeletal muscle. Front Genet. 2019;10:91.

Zhang K, Li Q, Kang X, Wang Y, Wang S. Identification and functional characterization of IncRNAs acting as ceRNA involved in the malignant progression of glioblastoma multiforme. Oncol Rep. 2016;36:2911-25.

Zhang J, Liu L, Li J, Le TD. LncmiRSRN: identification and analysis of long noncoding RNA related miRNA sponge regulatory network in human cancer. Bioinformatics. 2018;34:4232-40.

Zhang Y, et al. Lnc-PDZD7 contributes to stemness properties and chemosensitivity in hepatocellular carcinoma through EZH2-mediated ATOH8 transcriptional repression. J Exp Clin Cancer Res. 2019;38:92.

Zhao Q, et al. Systematic comparison of hUC-MSCs at various passages reveals the variations of signatures and therapeutic effect on acute graft-versushost disease. Stem Cell Res Ther. 2019;10:354.

\section{Publisher's Note}

Springer Nature remains neutral with regard to jurisdictional claims in published maps and institutional affiliations.
Ready to submit your research? Choose BMC and benefit from:

- fast, convenient online submission

- thorough peer review by experienced researchers in your field

- rapid publication on acceptance

- support for research data, including large and complex data types

- gold Open Access which fosters wider collaboration and increased citations

- maximum visibility for your research: over $100 \mathrm{M}$ website views per year

At BMC, research is always in progress.

Learn more biomedcentral.com/submissions 